\title{
PERFORMANCE IMPROVEMENT OF THE KEK PS-BOOSTER SYNCHROTRON
}

\section{YAMANE, E. TAKASAKI, S. HIRAMATSU, N. KUMAGAI and M. KIHARA KEK, National Laboratory for High Energy Physics Oho 1-1, Tsukuba-shi, Ibaraki-ken, 305 Japan}

The beam intensity of the KEK PS-Booster synchrotron cleared the level of $2 \times 10^{12} \mathrm{ppp}$. To realize this intensity many efforts have been payed not only to recover missed Booster ring acceptance and to make the Booster RF power supply tolerable against a heavy beam loading, but also to tune synthetically all the system from the ion source to the $12 \mathrm{GeV}$ synchrotron.

\section{Introduction}

At the KEK proton accelerator [1], a project has been undertaken for the last two years to increase beam intensity of the booster synchrotron to $2 \times 10^{12} \mathrm{ppp}$ from $8 \times 10^{11} \mathrm{ppp}$ at the start. The aim has been recently achieved and the synchrotron is now operated in routine at an intensity level of $2 \times 10^{12} \mathrm{ppp}$. In the first year, many efforts were payed to understand and solve two problems, namely a low injection efficiency below $90 \%$ and a large beam loss about 1 msec after injection. These problems were settled in a year. At the beginning of 1988 , the injection efficiency became nearly $100 \%$ and the beam loss disappeared. Then the beam intensity was about $1.2 \mathrm{x}$ $10^{12} \mathrm{ppp}$. However, it steadily increased acompanying progress of injector tuning and inprovement of the RF power supply to tolerate higher beam intensity.

\section{Present Status of the Booster Synchrotron}

At present, the KEK PS-Booster synchrotron accelerates about $2 \times 10^{12}$ protons upto $500 \mathrm{MeV}$ at a repetition rate of $20 \mathrm{~Hz}$ and the average current is $6 \mu \mathrm{A}$ in the routine operation. For the BSF

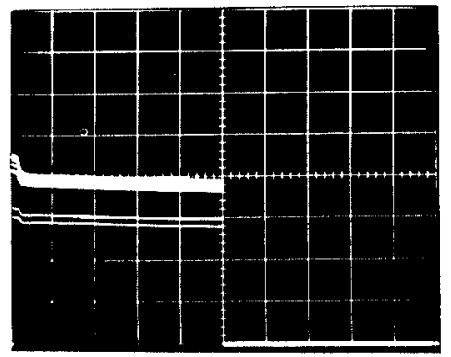

(a) before improvement

$$
\begin{aligned}
& 2 \times 10^{11} \mathrm{ppp} / \mathrm{div} \\
& 5 \mathrm{msec} / \mathrm{div}
\end{aligned}
$$

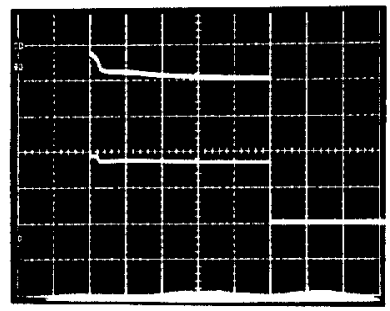

(b) after improvement

$$
\begin{aligned}
& 5 \times 10^{11} \mathrm{ppp} / \mathrm{div} \\
& 5 \mathrm{msec} / \mathrm{div}
\end{aligned}
$$

Fig. 1 Improvement of the Booster beam intensity during acceleration
(Booster Synchrotron Utilization Facility) this full beam is usually supplied, but a reduced amount of proton is supplied for the $12 \mathrm{GeV}$ main ring, because the beam intensity which can be safely accelerated to $12 \mathrm{GeV}$ is limitted to about $4 \times 10^{12}$ by beam losses at the beginning of acceleration and at the transition energy. A present typical time dependence of the Booster beam intesity is shown in Fig. 1 (b) with that at the start of the intesity improvement project (a).

The efficiency of $\mathrm{H}^{-}$injection is now nearly $100 \%$ even when about $2.4 \times 10^{12} \mathrm{H}^{-}$ions are injected. The beam loss at about 1 msec after injection which had been a serious question for a long time is now supressed upto an intensity level of more than $1.7 \times 10^{12}$ ppp.

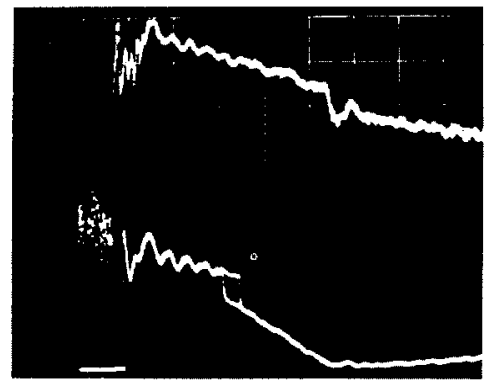

(a) before improvement upper: phase error signal lower : $\Delta R(10 \mathrm{~mm} / \mathrm{div}, 0.2 \mathrm{msec} / \mathrm{div})$

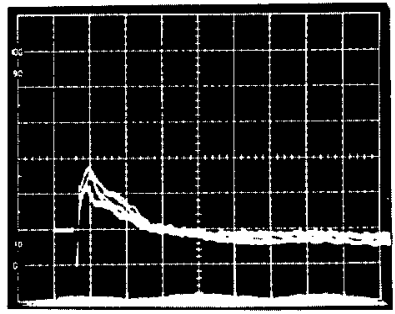

(b) $\Delta \mathrm{R}$ after improvement ( $5 \mathrm{~mm} / \mathrm{div}, 0.2 \mathrm{msec} / \mathrm{div}$ )

Fig. 2 Oscilloscope displays showing reduction of the $\Delta R$ just after injection

\section{$\underline{\text { Readjustments }}$}

\section{Recovery of missed acceptance}

In some machine studies for the project to upgrade the Booster beam intensity, it was found that the orbit radius was considerably large at the injection time. The $\Delta \mathrm{R}$ ( error of the orbit radius ) was about $20 \mathrm{~mm}$ or more as is shown in Fig. 2 (a) lower. The $\Delta R$ was coupled with the size of the injection bump orbit [2] as Fig 3 shows. Therefore both increase of the minimum guiding field 


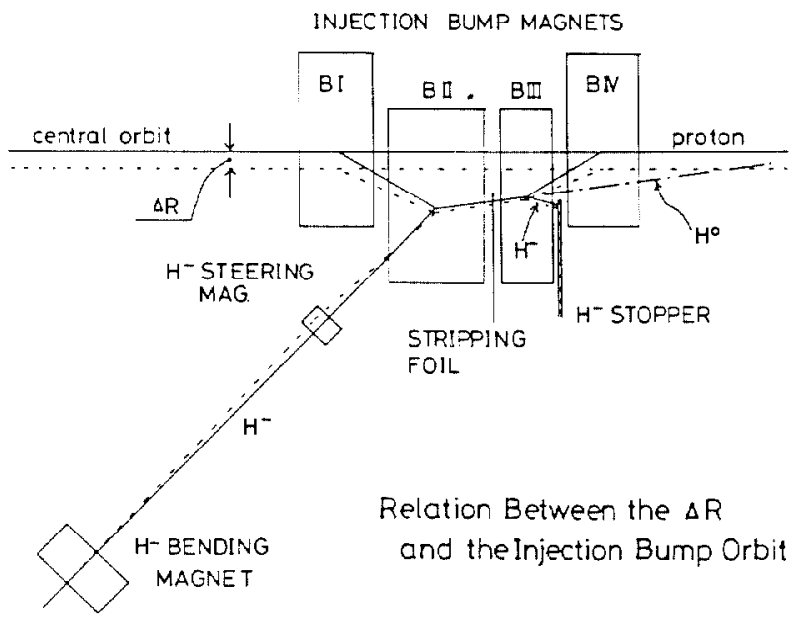

Fig. 3 Schematic view of the beam orbits around the Booster injection point

and reformation of the bump orbit were necessary to correct the $\Delta R$. After the correction the $\Delta R$ became fairly small as Fig. 2 (b) shows and the horizontal acceptance seemed to recover a considerable amount.

In the vertical plane, a correction of the closed orbit was made with a pair of steering magnets which were newly installed in two straight sections. Although these steering magnets are d.c. magnets, they are effective to correct the closed orbit at the injection time. Figure 4 shows Booster beam intensity contours on the plane of steering magnet currents. At present they are operated with $\mathrm{I}_{1}=$ $4.8 \mathrm{~A}$ and $\mathrm{I}_{2}=0.0 \wedge$

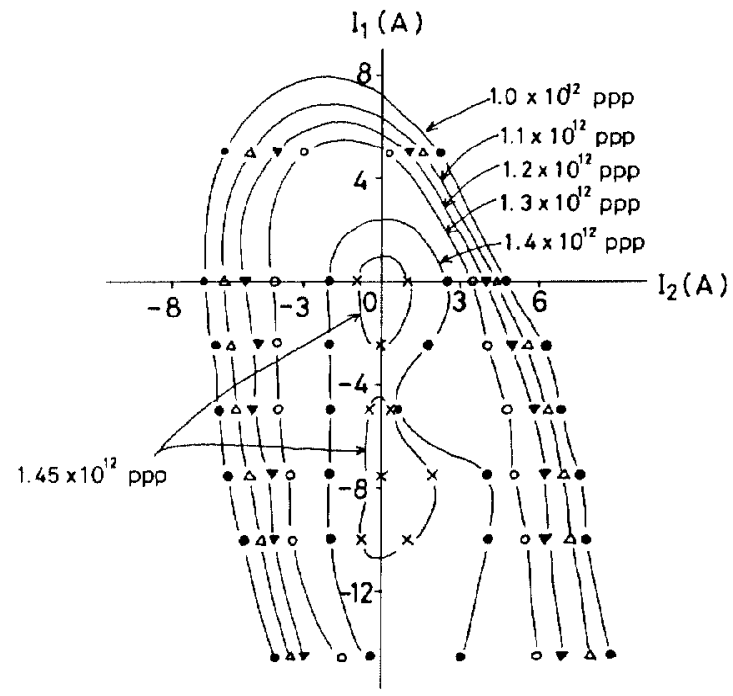

Fig. 4 Booster beam intensity contours on the plane of steering magnet currents

\section{Improvements of RF Power Supply}

The bias power supply in the Booster RF power supply II was improved so that its frequency response sufficiently covered the frequency range $(<6 \mathrm{kHz}$ ) of the synchrotron oscillation at the injection time. Figure 5 shows the frequency response before (a) and after (b) improvements. It effectively suppressed the fluctuation of the RF bucket caused by the large fluctuation of the autotune signal which had occured accompanying the beam injection.

Also improvements in connection between ground levels of the power amplifier and the cavity made equality of those levels much surer and greatly suppressed the misoperation of the autotune phase detector. This considerably raised tolerability of the RF power

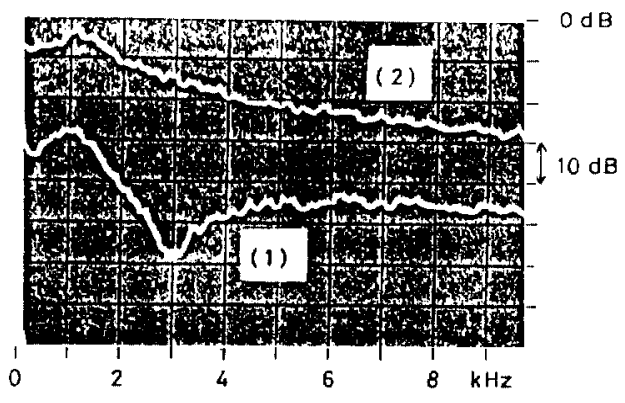

(a) before improvement (May, 1987)

(1) output current amplitude

(2) input for the driver circuit when the bios current is $500 \mathrm{~A}$

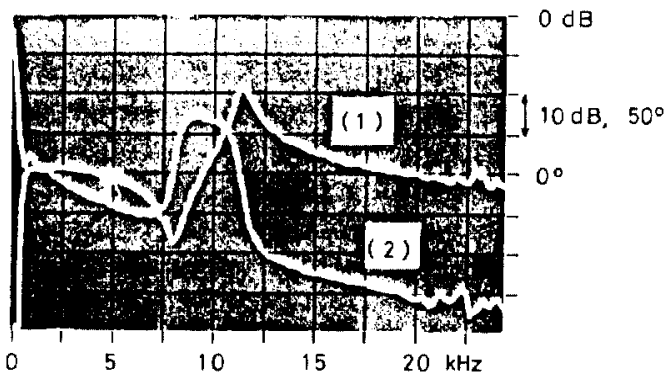

(b) after improvement (Jan, 1988)

(1) output current amplitude

(2) output current phose

Fig. 5 Frequency response improvement of the Booster RF Il ferrite bias power supply

supply to higher beam intensity.

In addition to these improvements, it seems very effective for improvement of adiabatic beam capture to have changed the beam injection time from $240 \mu \mathrm{sec}$ after to $200 \mu \mathrm{sec}$ before the bottom of the guiding field.

\section{Power Level Readiustment of two linac tanks}

Since the addition of the tank II to upgrade the $\mathrm{H}^{-}$beam energy $1040 \mathrm{MeV}$ [3], tank power levels had been set at about 1.4 MW for the tank I and at about $1.05 \mathrm{MW}$ for the tank II, following former experiences with the $20 \mathrm{MeV}$ linac and early tuning results of the new $40 \mathrm{MeV}$ linac. However, it turned out at around July, 1987

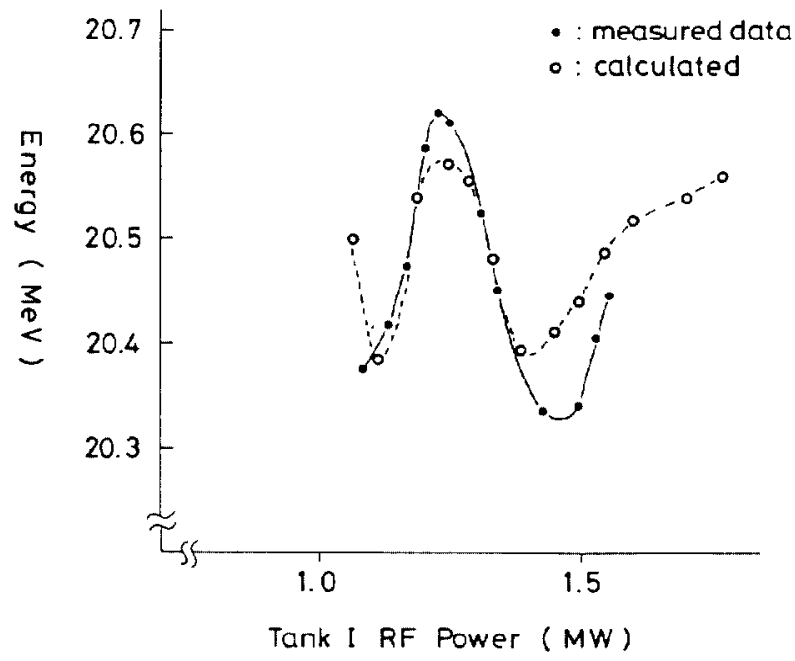

Fig. 6 Tank I RF power dependence of the output beam energy 


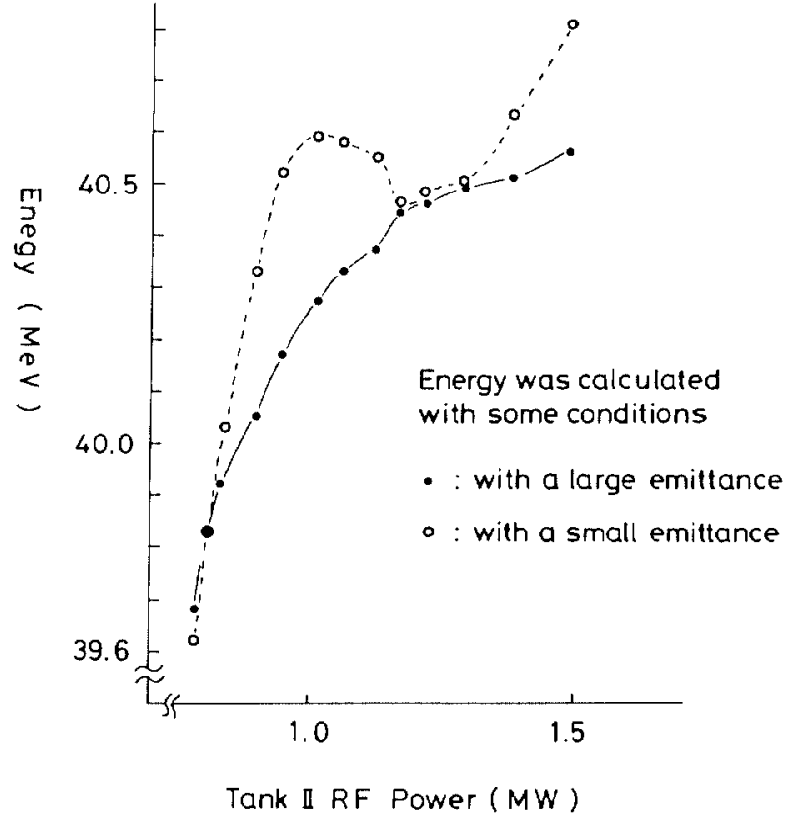

Fig. 7 Tank II RF power dependence of the output beam energy

that, when the power level of the tank I got down to $1.2 \mathrm{MW}$, the booster injection efficiency became considerably high and the beam loss at $1 \mathrm{msec}$ after injection decreased despite of rather high beam intensity. The value 1.2 MW meant that the energy of output beam from the tank I was $20.6 \mathrm{MeV}$, as is shown in Fig. 6. And 20.6 $\mathrm{MeV}$ was the design value of the injecting energy for the tank II.

The power level of the tank II was gradually raised from the autumn of 1987 and also settled at around 1.1 MW. Following the design calculation, the fact that the power level of the tank II was about 1.1 MW meant that the energy of output beam from the tank II was nearly $40.4 \mathrm{MeV}$ as is shown in Fig. 7. This energy was slightly higher than $39.5 \mathrm{MeV}$ to which the $\mathrm{B}_{\min }$ (minimum of the guiding field) had been adjusted in the early tuning after the linac upgrade. It explained naturally why the $B_{\min }$ had to be raised to reduce the $\Delta R$ just after injection.

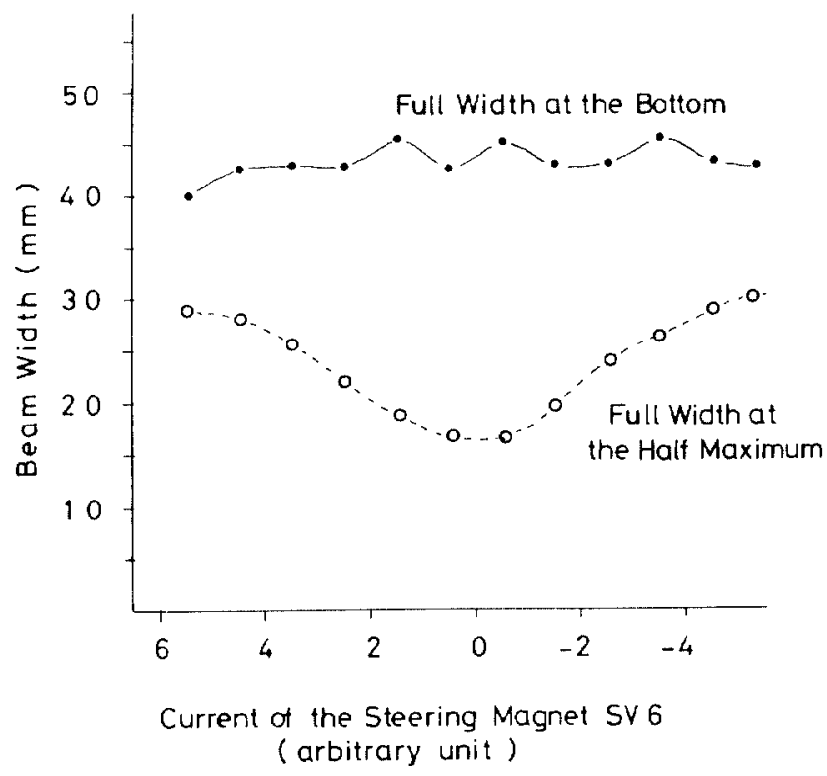

Fig. 8 Dependence of the vertical beam width at a profile monitor Pr-5 in the Booster extraction line upon current of a steering magnet SV-6 in the Booster injection line

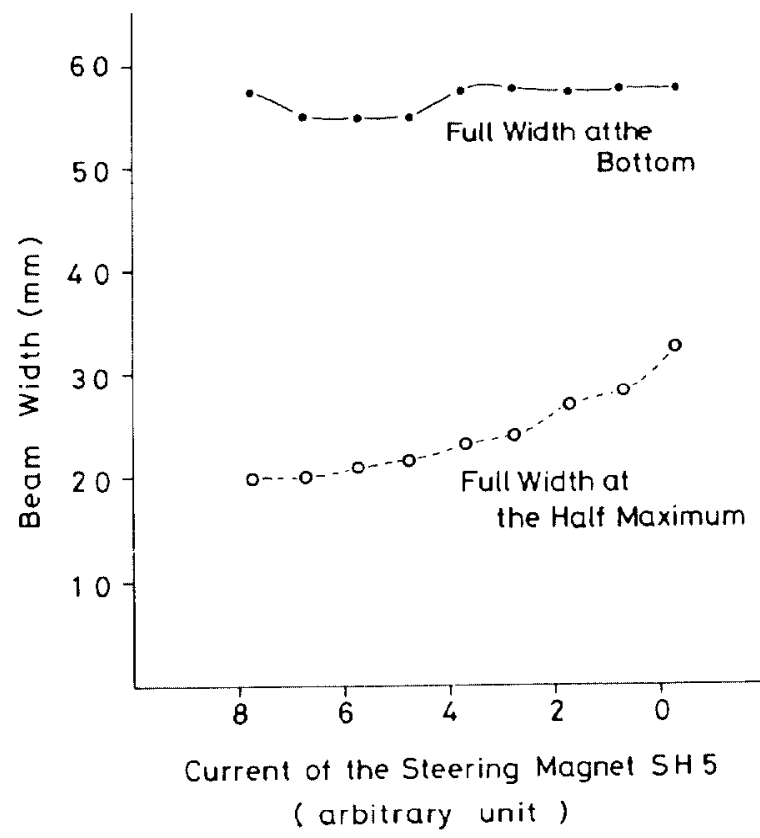

Fig. 9 Dependence of the horizontal beam width at a profile monitor Pr-6 in the Booster extraction line upon current of a steering magnet $\mathrm{SH}-5$ in the Booster injection line

\section{Qverall Tuning from the Ion Source to the Main Ring}

In the first year of the project, it was found that some profile monitors in the beam transport line from the booster to the main ring provided convenient and effective monitors to observe injection error of the booster synchrotron. Figures 8 and 9 show examples of beam size variation depending upon the current of steering magnet in the Booster injection line. By this method of monitoring, injection error of the Booster became to be considerably suppressed.

In the next year, more comprehensive tuning from the $\mathrm{H}^{-}$ion source to the Main ring was undertaken. Accompanying progress of the tuning, conditions of the Booster injection and acceleration were obviously improved. Especially tuning of the linac injection line was very effective. In this situation the Booster beam intensity was steadily increased as the beam intensity tolerance of the RF power supply became higher.

\section{Acknowledgement}

It is definitely acknowledged that all achievements described here were due to the patient efforts of all PS group members and the authors were speakers at the second meeting to discuss intensity upgrade of the Booster which became the base of this report.

\section{$\underline{\text { References }}$}

[1] M. KIHARA et al., Proceedings of the International Workshop on Hadron Facility Technology, Santa Fe, Inn at Loretto, USA, Feb 2-5, 1987. KEK Preprint 86-107, February 1987, A

KEK PS Group ( Presented by 1. YAMANE), Proceeding of the 6th Symposium on Accelerator Science and Technology, October 27-29, 1987, p36

[2] T. KAWAKUBO et al, Proceedings of the 13th International Conference on High Energy Accelerators, Novosibirsk, USSR, August 7-11, 1986, p286-289

I. YAMANE and H. YAMAGUCHI, Nuclear Instruments and Methods in Physics Research A254 (1987) 225-228

T. KAWAKUBO, Nuclear Instruments and Methods in Physics Research A265 (1988) 351-363

[3] S. FUKUMOTO et al., Particle Accelerator, Vol, 20, No.1 (1987), p71

S. FUKUMOTO et al.,1986 Linear Accelerator Conference Proceedings, SLAC, Stanford, CA, USA, June 2-6, 1986, 116-118

T. KATO and S. MACHIDA, 1986 Linear Accelerator Conference Proceedings, SLAC, Stanford, CA, USA, June 2$6,1986,119-121$ 